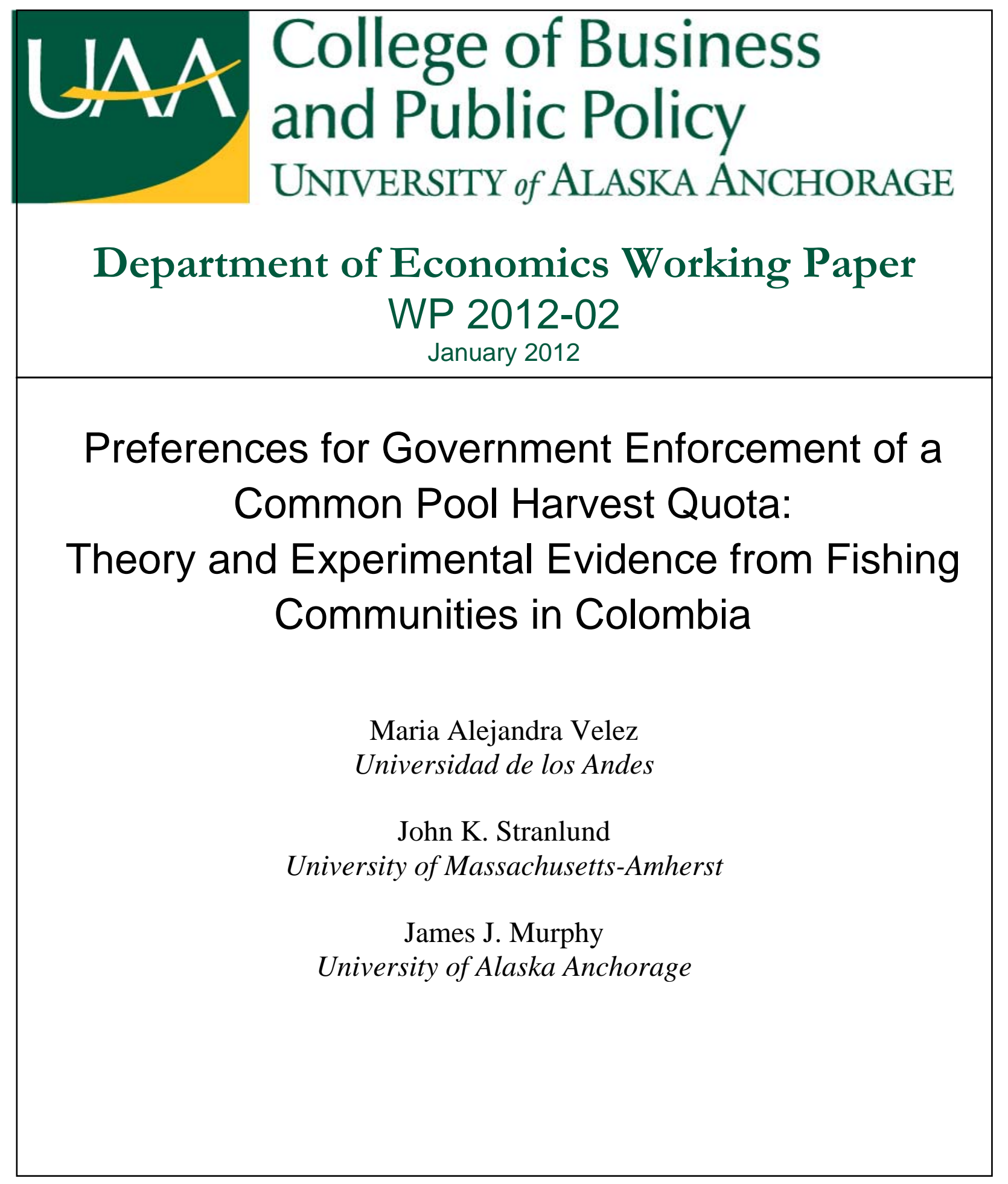

UAA DEPARTMENT OF ECONOMICS

3211 Providence Drive

Rasmuson Hall 302

Anchorage, AK 99508

http://econpapers.uaa.alaska.edu/ 


\title{
Preferences for Government Enforcement of a Common Pool Harvest Quota: Theory and Experimental Evidence from Fishing Communities in Colombia
}

\author{
MARIA ALEJANDRA VELEZ ${ }^{*}$ \\ School of Management, Universidad de los Andes, Bogotá, Colombia. \\ mav@adm.uniandes.edu.co \\ JOHN K. STRANLUND \\ Department of Resource Economics, University of Massachusetts-Amherst, Amherst, MA 01003 \\ stranlund@isenberg.umass.edu \\ JAMES J. MURPHY \\ Department of Economics, University of Alaska Anchorage, Anchorage, AK 99508 \\ murphy@uaa.alaska.edu
}

\section{Acknowledgements}

We are grateful to Maria Claudia Lopez and members of the Faculty of Environmental and Rural Studies at Javeriana University in Bogotá, Colombia, who provided outstanding support for our fieldwork. The experiments would not have been possible without the assistance of local community leaders who helped the research team develop credibility with local community members. We are also indebted to WWF Colombia for coordinating the fieldwork in the Pacific region. Financial support from the U.S. Embassy in Bogotá is gratefully acknowledged. We assume complete responsibility for the final contents of this article.

\footnotetext{
${ }^{*}$ Corresponding author
} 


\title{
Preferences for Government Enforcement of a Common Pool Harvest Quota: Theory and Experimental Evidence from Fishing Communities in Colombia
}

\begin{abstract}
We examine individual harvesters' preferences for government enforcement of a quota imposed on the exploitation of a common pool resource. We develop a model of Nash behavior by identical risk neutral harvesters to explain individual equilibrium preferences for enforcement of an efficient harvest quota. If the quota is not enforced well, we demonstrate that individual harvesters will always prefer increased enforcement—either increased monitoring or increased penalties_ of the quota. We conduct a test of this theoretical result with data from framed common pool resource experiments conducted in artisanal fishing communities in three regions of Colombia. Subjects were given the opportunity to express their preferences for enforcement by voting on two levels of enforcement of a harvest quota, with and without communication. The two enforcement strategies involved the same probability that the government would audit individual harvesters, but differed in the level of the penalty for noncompliance. Contrary to theory, individuals voted for the lower inefficient penalty about $80 \%$ of the time and groups implemented this weaker enforcement strategy over $90 \%$ of the time. Giving subjects the opportunity to vote on the enforcement strategy did not lead to more efficient harvests, nor did allowing subjects to communicate before voting.
\end{abstract}

JEL Classifications: C93, Q20, Q28

Keywords: common pool resources, field experiments, enforcement, regulation, voting 


\section{Introduction}

The standard externality resulting from the exploitation of common pool fisheries (and other common pool resources) is that individual harvests increase the costs of other harvesters. Consequently, noncooperative and unregulated exploitation of a fishery is generally inefficient. In many cases, therefore, government regulation of common pool resources is justified. Of course, all regulations must be enforced. Thus, the efficient design of a common pool regulation must include efficient enforcement strategies to counteract harvesters’ incentives to violate a regulation and to account for the costs of enforcement. However, many fisheries regulations are poorly enforced (Dolsak and Ostrom 2003), especially in the developing world. Potential reasons include the unwillingness of authorities to allocate adequate resources to enforcement and the lack of institutional capacity.

In this paper we analyze, both theoretically and with experimental evidence, one aspect of weak enforcement of common pool fisheries: namely, do individual harvesters prefer stricter enforcement of fishery regulations? This is an important issue because individual harvesters may have opportunities to influence and participate in the design and implementation of regulations, and hence, to express their preferences for weaker or stronger enforcement. Stronger enforcement of a regulation involves a fundamental tradeoff for the individual harvesterstronger enforcement increases the expected costs of noncompliance for an individual, but encourages lower aggregate harvests so that the cost externality associated with harvesting from a common pool is reduced. Which effect dominates will determine whether individual harvesters prefer stronger or weaker enforcement.

Our motivation for studying this aspect of weak enforcement of fisheries regulations comes from our interest in the efficacy of government regulations of artisanal fisheries in the 
developing world. Enforcement of such regulations is typically quite weak. Thus, our primary interest is whether small-scale harvesters in a regulated fishery prefer that the government exert more enforcement pressure on these fisheries or if weak enforcement is consistent with their desires.

We begin by developing a theoretical model of identical risk neutral harvesters of a common pool resource to explain individual preferences for enforcement of an efficient harvest quota. We derive an optimal harvest regulation that consists of a harvest quota and a costly monitoring strategy to enforce the quota, given a fixed unit penalty for noncompliance. We then suppose that the quota is imperfectly enforced in the sense that monitoring is not sufficient to induce full compliance with the quota, and determine whether individual harvesters prefer increased enforcement. We demonstrate that individual harvesters will always prefer increased enforcement—either increased monitoring or increased penalties_of the quota. The reason is that stricter enforcement leads to lower aggregate harvests, which benefits an individual harvester more than the increase in his or her expected costs of noncompliance.

We test this theoretical result with data from framed common pool resource experiments conducted in three geographically-distinct artisanal fishing communities in Colombia with subjects whose livelihoods depend upon successful management of a shared resource. Subjects in some sessions were given the opportunity to express their preferences for enforcement by voting on two levels of the marginal penalty for violating a harvest quota, one low and the other significantly higher. The harvest quota and the probability that the government would audit individual harvesters were constant for all the treatments. Our theoretical model predicts that the subjects would always vote for the higher penalty, make more conservative harvest choices, and be better off than if the regulation were enforced with the lower penalty. 
Contrary to this prediction, subjects and groups were rarely willing to implement the more efficient penalty. In the absence of communication, individuals voted for the higher penalty only about $20 \%$ of the time, and as a result, groups implemented this stricter enforcement strategy through majority rule less than $10 \%$ of the time. This finding is robust across the three regions. Moreover, giving subjects this opportunity to participate in part of the design of a regulation by voting on the quota violation penalty did not improve the efficiency of harvest choices. $^{1}$

The experimental literature on participation in the design of policies to promote more efficient choices in social dilemmas is mixed. While some researchers have found that voting on certain elements of policies can increase cooperation in these settings, ${ }^{2}$ this is rarely the case for subject participation in the design of enforcement. In a common pool resource experiment, Vyrastekova and van Soest (2003) asked subjects to vote on whether to allow an enforcer (an experiment participant) to keep the fines when taking violators to court. An affirmative vote was the efficient choice; however, they found that harvesters infrequently voted to allow the enforcer to keep these penalties, leading to inefficient outcomes. Bischoff (2007) also found that allowing subjects to vote on rules did not lead to improved outcomes. Bischoff's main conclusion is that although subjects often voted for efficient quotas, they were reluctant to support increased monitoring intensity. Consequently, groups that could change inefficient regulations through majority rule voting actually performed worse than groups who were not given this opportunity.

\footnotetext{
${ }^{1}$ In many common pool field experiments like ours, subjects tend to be more conservative than predicted by models of purely self-interested harvesters (e.g., Cardenas et al. 2000; Velez et al. 2009). We find this result in our study as well.

${ }^{2}$ For example, both Walker et al. (2000) and Margreiter et al. (2005) find that voting on the allocation rules for a common pool resource substantially increased efficiency relative to a baseline with no opportunity for collective choice. Similarly, voting on the possibility of rewarding or punishing other group members tends to increase cooperation (Ostrom et al., 1992; Sutter et al., 2010).
} 
In a public goods experiment, Tyran and Feld (2006) compare the effects of an exogenously-imposed regulation that required each person to contribute all of her endowment to the public good vis-à-vis allowing subjects to vote on the implementation of this regulation. In both scenarios, the regulation was backed by perfect monitoring and a penalty for all violations of this requirement. Their main conclusion is that compliance is significantly greater when the subjects voted to implement a regulation with a sanction that was too low to induce full compliance. Our experiments, as well as those of Vyrastekova and van Soest (2003) and Bischoff (2007), are fundamentally different from Tyran and Feld's because their subjects voted on whether to impose a regulation. In our work, subjects voted on the severity of sanctions, given that a regulation would be imposed. Thus, Tyran and Feld do not address the question of individual desires for increased enforcement that is the goal of our study. Moreover, the penalty in Tyran and Feld's work is imposed with certainty in cases of noncompliance, whereas our penalties are imposed randomly because monitoring to detect noncompliance is imperfect. ${ }^{3}$ These authors took a similar approach in a separate experimental study of tax compliance and found similar results; specifically they found that compliance was higher when subjects voted to accept a certain fine for noncompliance than when the fine was exogenously imposed (Feld and Tyran 2002).

The study that is closest to ours is by Alm et al. (1999) who studied voting on the enforcement parameters of an income tax policy (tax rate, audit rate and fine). They also found that subjects were unwilling to vote for stricter enforcement. When subjects could not communicate, they always voted against stricter enforcement, both increases in audit

\footnotetext{
${ }^{3}$ Cardenas' (2005) experiments are similar to Tyran and Feld's (2006), although he focused on a common pool resource environment and his main objective was to examine possible differences in the decisions made by university students in Bogotá, Colombia and field subjects in rural areas of Colombia. He finds that, in the majority of the cases, participants in rural areas opposed external regulation but students in urban areas normally voted in favor of regulation.
} 
probabilities and increases in the fine for evaded taxes, even in treatments in which it was efficient to do so. The subjects sometimes voted for increased enforcement when they could communicate with one another. Moreover, when the subjects voted for weaker enforcement, compliance rates decreased significantly. Alm et al. (1999) suggest that voting against stronger enforcement sends a signal of social acceptability of tax evasion.

In summary, while other researchers have found that subject participation in the decision about whether to enforce an external regulation may lead to more efficient choices (Tyran and Feld 2006), it is clear that participation in the decision about the level of enforcement does not (Alm et al. 1999). We come to the same conclusion, but our work is the first to examine this issue in a field setting. The subjects in our experiments are mainly fishermen who operate under poorly enforced regulations or are intimately connected to a local fishery in other ways. Thus, the overexploitation of common pool resources and the (in)effectiveness of government regulations designed to promote efficient harvests are critically important to the subjects in our experiments. Moreover, we exploit the heterogeneity of the field by conducting our experiments in three regions of Colombia in communities that are dependent on a local fishery, but that vary in other ways. In each area we find the same general result: subjects are unwilling to vote for stricter regulatory enforcement, even when it would be in their best interests (both individually and collectively) to do so. Thus, we demonstrate that results obtained in laboratory experiments about the inefficiency of allowing subjects to vote on enforcement extend to artisanal fishing communities in Colombia. 


\section{Individual preferences for enforcing a common pool harvest quota}

Our experimental design is based on a model of behavior by risk neutral harvesters who face a harvest quota that they may violate. The model is a static one that is similar to the model developed by Cornes and Sandler (1983), and used in experimental work by Ostrom et al. (1994), among others. A fixed group of $n$ identical risk neutral individuals harvest a common pool resource. Individual $i$ harvests $x_{i}$ units, which sell at a constant price $p$. The individual's harvest costs are $c \sum_{i=1}^{n} x_{i}+d x_{i} \sum_{i=1}^{n} x_{i}$, where $c$ and $d$ are positive constants. By defining $x_{-i}=\sum_{j \neq i} x_{j}$, we can write $i$ 's harvest costs more compactly as $c\left(x_{i}+x_{-i}\right)+d x_{i}\left(x_{i}+x_{-i}\right)$.

These components of the cost function capture the social dilemma of the model: $d x_{i}\left(x_{i}+x_{-i}\right)$ captures the cost externality that is typical of common pool problems, while $c\left(x_{i}+X_{-i}\right)$ captures negative externalities that reduce individual existence or non-use values. The individual has an endowment $e_{i}$.

Imagine that a harvest quota has been imposed in the common pool resource. Suppose further that this quota has been set at the level of individual harvests that maximizes the joint payoffs of the harvesters less the aggregate costs of enforcing the quota. Denote the harvest quota as $x^{e}$, and note that it must be a uniform quota because the harvesters are identical. To enforce this quota the authorities monitor the harvesters and impose a penalty when one is found to have taken more from the common pool than the quota allows. Let the probability that a violation by an individual harvester is detected be $\pi$ and let this probability be the same for each harvester. The unit penalty for a violation is $\phi$ so that an individual with violation $x_{i}-x^{e} \geq 0$ 
faces a penalty $\phi\left(x_{i}-x^{e}\right) \geq 0$ if the violation is discovered. The unit penalty is high enough to allow the regulation to involve complete compliance if this turns out to be optimal.

Each individual harvester chooses a harvest level to maximize his expected payoff,

$$
w_{i}=e_{i}+p x_{i}-c\left(x_{i}+x_{-i}\right)-d x_{i}\left(x_{i}+x_{-i}\right)-\pi \phi\left(x_{i}-x^{e}\right),
$$

subject to $x_{i} \geq x^{e}$ to reflect the fact that an individual never has the incentive to be overcompliant. It is straightforward to show that the unique symmetric Nash equilibrium harvests $\operatorname{are}^{4}$

$$
x^{n}(\pi, \phi)=\frac{p-c-\pi \phi}{d(n+1)}
$$

Assume throughout that $p-c-\pi \phi>0$ so that Nash harvests are always strictly positive. Using [1], individual equilibrium payoffs are

$$
w\left(\pi, \phi, x^{e}\right)=e+p x^{n}(\pi, \phi)-n c x^{n}(\pi, \phi)-d n\left(x^{n}(\pi, \phi)\right)^{2}-\pi \phi\left(x^{n}(\pi, \phi)-x^{e}\right) .
$$

Now let us determine the optimal harvest regulation. The optimal harvest quota $x^{e}$ and optimal detection probability $\pi^{e}$ maximize the net value of harvests from the common minus the costs of monitoring, taking into account how the enforcement variables determine equilibrium harvests. Let the cost of establishing the optimal detection probability for each firm be $m \pi^{e}$. If optimality calls for noncompliance with the quota, penalties are treated as mere transfers so that the government ignores them. Therefore, the regulatory objective is to choose $x^{e}$ and $\pi^{e}$ to maximize

$$
W\left(\pi^{e}, x^{e}\right)=n\left\{e+p x^{n}\left(\pi^{e}, \phi\right)-n c x^{n}\left(\pi^{e}, \phi\right)-d n\left(x^{n}\left(\pi^{e}, \phi\right)\right)^{2}-m \pi^{e}\right\}
$$

\footnotetext{
${ }^{4}$ All derivations of the results in this section are available from the authors upon request.
} 
subject to $\pi^{e} \in[0,1]$ and $x^{e} \leq x^{n}\left(\pi^{e}, \phi\right)$ to again reflect the fact that harvesters will never find it optimal to over-comply.

Note that the harvest quota does not enter the authority's objective function [4]. This implies that there is no loss or gain to setting the quota so that the harvesters are fully compliant; that is, $x^{e}=x^{n}\left(\pi^{e}, \phi\right) .{ }^{5}$ Once this constraint is imposed on the authority's problem, it is straightforward to show that the optimal detection probability is

$$
\pi^{e}=\frac{(p+n c)(n-1) \phi-m d(n+1)^{2}}{2 n \phi^{2}}
$$

as long as the right side of [5] is an element of [0,1] . If the right side is negative, then $\pi^{e}=0$, which indicates that it is not worth regulating the common. This may occur if the marginal cost of monitoring $m$ is sufficiently high. On the other hand if the right side of [5] is greater than one, then $\pi^{e}=1$, indicating perfect monitoring. We ignore these possible boundary solutions. Then, substituting [5] into $x^{e}=x^{n}\left(\pi^{e}, \phi\right)$ yields the optimal harvest quota:

$$
x^{e}=x^{n}\left(\pi^{e}, \phi\right)=\frac{(p-n c) \phi+m d(n+1)}{2 d n \phi} .
$$

Note that setting the marginal cost of monitoring $m$ equal to zero yields the first-best harvest quota. Positive marginal monitoring costs imply that the second-best quota [6] is higher than the first-best quota.

For many regulations, however, enforcement strategies are not designed to induce perfect compliance to a standard. Imperfect compliance in our theoretical model results if $\pi<\pi^{e}$. In fact the question we are most interested in this paper is whether individuals prefer increased

\footnotetext{
${ }^{5}$ Part of the indifference the authority has toward compliance vs. noncompliance has to do with our assumption that penalties have no real effects. However, authorities typically have to expend resources to impose and collect penalties from violators. Incorporating the assumption that collecting penalties is costly in our model would yield the result that it is unambiguously optimal to enforce full compliance to the quota.
} 
enforcement when an efficient quota is imperfectly enforced. To answer this question return to an individual's payoff in a symmetric Nash equilibrium given by [3]. Into [3] substitute [6] for $x^{e}$ and [2] for $x^{n}(\pi, \phi)$ to obtain an individual's payoff as a function solely of the enforcement parameters, $\pi$ and $\phi$. Then it is straightforward to calculate:

$$
\begin{aligned}
& \frac{\partial w(\pi, \phi)}{\partial \pi}=\frac{d m(n+1)^{3}+\phi\left[(p+n c)(n-1)^{2}+4 n \pi \phi\right]}{2 d n(n+1)^{2}}>0 ; \\
& \frac{\partial w(\pi, \phi)}{\partial \phi}=\frac{\pi\left[(p+n c)(n-1)^{2}+4 n \pi \phi\right]}{2 d n(n+1)^{2}}>0 .
\end{aligned}
$$

Since both of these derivatives are strictly positive, each individual harvester is better off with stricter enforcement of the efficient harvest quota. ${ }^{6}$

It is the nature of the social dilemma that all common pool users are better off if they restrict their harvests, but individually they have little incentive to do so. A regulation can align individual and group incentives, but it must be enforced well to be efficient. However, even a poorly enforced regulation that is widely violated can improve the welfare of harvesters if it motivates them to restrict their take from the common pool. The regulation will not be efficient, but it could be an improvement. Now, an individual harvester that violates a quota would never prefer more stringent monitoring or higher sanctions directed solely at him. However, our results reveal that the harvester and all others prefer stricter enforcement if it is imposed on the entire group. The reason is simple: enhanced enforcement pushes aggregate harvests toward the efficient level, which produces a gain for each harvester that is greater than the increase in their individual expected sanctions for noncompliance.

\footnotetext{
${ }^{6}$ In fact, it can be shown that harvesters will prefer increased enforcement even when they have to pay for it. A proof of this assertion is also available from the authors.
} 


\section{Experimental Design}

We test the theoretical result that harvesters prefer stricter enforcement with data from framed common pool resource experiments conducted in three regions of Colombia. We framed the experiments an individual harvesting decision from a local fishery. ${ }^{7}$ To examine the robustness of our results, all experimental treatments were conducted in each of the three regions. These areas are similar in that each depends heavily on local fisheries—in fact, most of the participants in our experiments were fishermen—but they differ in many other ways. We do not pose hypotheses about how behavior may differ across regions, mainly because it is not possible to conduct rigorous tests with only three regions. However, a brief description of how the regions are different is appropriate. Summary statistics of the subjects' characteristics are provided in Table 1.

Subjects living near the Ensenada de Tumaco in the Pacific region are members of AfroColombian communities who, for the most part, live in collective territories. The participants in the Magdalena region are from the towns of La Dorada, Caldas, and surrounding villages, and are part of a mostly white and mestizo population that harvests fish from the Magdalena River and the adjacent lake, Charca de Guarinocito. In terms of basic characteristics, the participants here were similar to those in the Pacific region. The final set of experiments was conducted near the city of Santa Marta in the Caribbean region. Subjects there are part of a multiethnic population of whites, mestizos, African descendants, and indigenous people. The participants in this region are somewhat different than in the other two regions. The proportion of participants in this region who reported that fishing is their main economic activity is significantly lower than in the other two regions, as is the percent who report living in the area for more than 10 years.

\footnotetext{
${ }^{7}$ Using the Harrison and List (2004) taxonomy, these are framed field experiments because they are conducted with a population of subjects for which the phenomenon of interest to us (behavior in a common pool fishery) is also an important element of the subjects' experiences.
} 
Some of the other participants were small-scale fish buyers who then resold their product in Santa Marta. The rest were farmworkers. Subjects were also younger and more educated, with significantly fewer male subjects. See Velez et al. (2010) for a more thorough description of the communities.

Subjects were placed in groups of five and participated in a hand-run, 20-period common pool resource experiment that was framed as a harvest decision from a fishery. ${ }^{8}$ Each subject received an identical payoff table that was generated from a simple modification of [1], excluding the expected marginal penalty for violations, $\pi \phi$. The concept of zero harvest is difficult to explain in the field because the participants depend so critically on their use of local natural resources. Therefore, individual harvest choices were shifted by one to range from one to nine, rather than zero to eight. Accordingly, we modified [1] by defining $\hat{x}_{i}=x_{i}-1$ and created the individual payoff table from $\pi_{i}=e_{i}+p \hat{x}_{i}-c\left(\hat{x}_{i}+\hat{x}_{-i}\right)-d \hat{x}_{i}\left(\hat{x}_{i}+\hat{X}_{-i}\right)$, with parameters $p=$ 116.875, $c=17.875, d=2.75$, and $e_{i}=900 .^{9}$ In the experiments, individual harvests were constrained to integer values between one and nine units. In an unregulated environment the standard symmetric Nash equilibrium is achieved when each individual chooses to harvest seven units, while the individual harvest that maximizes group welfare is two units.

In every session, the first stage consisted of 10 rounds in an unregulated common pool resource environment without communication. The 10-round second stage distinguished the six treatments with the $3 \times 2$ factorial design summarized in Table 2 . A total of 360 individuals participated in the experiments, divided into 72 five-person groups ( 3 regions $\times 6$ treatments $\times 4$ groups per treatment).

\footnotetext{
${ }^{8}$ Assignment to groups was not completely random. We tried to ensure that relatives were in separate groups.

${ }^{9}$ Experiment instructions are available upon request.
} 
We consider two exogenously imposed enforcement strategies (Imposed Low and Imposed Medium) which consist of an individual harvest quota set at the level that maximizes a group's payoff $\left(x^{e}=2\right)$, but that differ with respect to the level of enforcement. ${ }^{10}$ Both enforcement strategies have the same $\pi=10 \%$ probability that a subject's harvest choice would be inspected. ${ }^{11}$ If an inspection revealed that a subject's harvest was greater than two, then that person incurred a financial penalty. For the Low penalty, the fine was $\phi^{L}=27$ pesos per unit above the quota. With this penalty, the resulting marginal expected penalty is not high enough to change the pure Nash strategy equilibrium from the unregulated equilibrium of seven units of harvest by each individual. For the Medium penalty, the fine was $\phi^{M}=165$ pesos per unit. The Nash strategy equilibrium with this penalty is reduced to six units of harvest for each individual. We chose enforcement strategies that could not support full compliance to the harvest quota, at least under a conventional theory of regulatory enforcement, because this is likely to be a characteristic of most regulatory controls of resource use in the developing world.

Each of the two voting treatments (Vote/Com and Vote/NoCom) allowed the subjects to vote on the size of the marginal penalty ( $\phi^{L}=27$ pesos or $\phi^{M}=165$ pesos), while keeping the same harvest quota and monitoring probability. Majority rule determined which penalty would be implemented. The vote took place at the start of each round. Participants were informed about the level of the penalty chosen by the majority and the distribution of the votes before deciding on their levels of harvest. The expected Nash equilibrium payoff of a risk neutral subject under

\footnotetext{
${ }^{10}$ We do not attempt to determine and implement the harvest quota that maximizes a group's payoff less monitoring costs, given by equation [8] in the previous section. To do so, we would have had to specify an ad hoc value for marginal monitoring costs $m$. Our hypothesis that individuals will always prefer increased enforcement is unaffected by this design choice.

${ }^{11}$ The results of inspections were not made public. In order to decide who in a group, if anyone, was inspected in a particular round, a ballot was chosen from a bag containing 5 ballots with the participants' numbers on them and 5 other blank ballots.
} 
the low penalty is 556.5 pesos, while it is 628 pesos under the medium penalty. Thus, as we have demonstrated theoretically, subjects are expected to always vote for the medium penalty.

Face-to-face verbal communication was permitted only in the three communication treatments. Every round, prior to making any decisions, subjects were given five minutes to discuss the experiment before returning to their seats and making their decisions in private.

After all subjects completed their decisions, the experimenter collected this information and announced to the group the aggregate level of harvest for that round. With this information individuals were able to calculate both the total harvest of the other four group members and their own individual payoffs. Individual earnings in the experiments ranged between 11,220 and 22,900 pesos with an average of 15,240 pesos (about US\$6.00). ${ }^{12}$ Earnings were paid in cash at the end of each experiment. Each experiment lasted about 3 hours. ${ }^{13}$

\section{Results}

\subsection{Voting choices}

Individual and group voting results for the two voting treatments are presented in Tables 3 and 4. Although our theoretical development produces the hypothesis that subjects would always vote for stricter enforcement of the two-unit harvest quota, the experiment results strongly suggest otherwise. Out of a total of 600 individual votes cast without communication (12 groups of 5 subjects over 10 rounds), only 119 (20\%) were for the medium penalty. Consequently, out of 120 opportunities (12 groups over 10 rounds), groups voted to implement the medium penalty less than $10 \%$ of the time. That subjects and groups voted against the medium penalty is consistent

\footnotetext{
${ }^{12}$ A day's wage in the regions where the experiments were conducted varied between 10,000 and 15,000 pesos at the time of the experiments.

${ }^{13}$ Before each experiment began, instructions were read aloud by the monitor and several practice rounds that did not count toward final earnings were played to familiarize the participants with the experiments. The real money rounds began after all participants understood the rules of the game.
} 
across regions. As shown in Table 3, in the absence of communication, individuals in the Caribbean voted for the medium penalty $7.5 \%$ of the time, in the Magdalena region 20.5\%, and in the Pacific region $31.5 \%$. Table 4 shows that, as a result of these individual votes, the medium penalty was never implemented in the Caribbean, in the Magdalena the medium penalty was implemented in only 4 of 40 opportunities, and in the Pacific only 5 of 40 opportunities.

Overall, communication did not change individual voting decisions by much and groups implemented the medium penalty only $16 \%$ of the time. Therefore, our main finding is that subjects and groups rarely voted to implement the more efficient medium penalty and this is robust across regions. These conclusions are consistent with the results of Bischoff (2007), Vyrastekova and van Soest (2003), and Alm et al. (1999), who also found that subjects were reluctant to impose stricter enforcement on their groups, even when it was efficient to do so.

\subsection{Effect of regulations on harvesting decisions in stage 2}

To examine the effects of the regulations in stage 2, we begin with the average harvests in Table 5. In the stage 1 no-regulation baseline, individual harvests averaged 5.6 in each of the three regions. Similar to other studies in this area, these average harvests are significantly below the Nash equilibrium prediction of seven units, but significantly above the efficient individual harvest of two units (Velez, et al. 2009 and 2010). ${ }^{14}$ Using a random effects Tobit model not reported in this paper, we confirm the results of Velez, et al. (2010) that (a) there is no statistically significant difference in the stage 1 harvest across the regions, and (b) for every treatment-region combination, individual harvests decisions in stage 2 are lower than those in the unregulated stage 1 Baseline. Thus, each of the second-stage institutions, regardless of whether

\footnotetext{
${ }^{14}$ Cardenas (2004) used a common pool design that is similar to ours and also found that participant's decisions in the field and in the lab tend to lie between their pure Nash strategies and the efficient harvest.
} 
the enforcement parameters were exogenously imposed or collectively agreed upon through a majority vote, was effective at inducing more conservative harvest choices vis-à-vis the first stage no enforcement baseline. This clearly suggests that the presence of an imperfectly enforced regulation that requires socially efficient choices induces more conservative harvest choices, regardless of whether subjects are able to vote on the regulation.

Although subjects rarely support the more stringent medium penalty, it is still possible that the ability to participate in the decision about the enforcement parameters could lead to more efficient harvest choices, even when the group collectively decides not to implement the medium penalty. To determine the effects on individual harvests of voting versus exogenously imposing an enforcement strategy we estimate the pair of random effects Tobit models in Table 6 that control for the censored nature of the data (individual decisions were constrained to be between 1 and 9) and the individual repeated observations. To control for changes in individual decisions over time, the first model in Table 6 includes only the data from the first five periods of stage 2, and the second model includes just the last five periods (stage 1 data are not included). The dependent variable is the individual harvest decision; the explanatory variables include dummy variables for the treatment effects by region, period effects (for stage two, the periods are numbered 11-20), and sociodemographic characteristics. The model excludes the constant, so the coefficients for the treatment $\times$ region dummy variables reflect individual harvest decisions after controlling for sociodemographic characteristics and time. Because of the small number of observations, the data do not include those instances in which groups voted to implement the medium penalty. Table 7 summarizes the Wald chi-squared tests of hypotheses related to both regional and treatment effects based on the models in Table 6 . 
Regional differences: As reported in Velez et al. (2010), and confirmed in Table 7, there are significant regional differences in individual harvests with the low penalty regardless of whether it was imposed or voted upon, or whether subjects were able to communicate with each other. Interestingly, there are significant regional differences in harvests with the imposed medium penalty in the first five periods of the second stage, but these differences are insignificant in the last five periods of the stage. Recall that the low penalty was chosen so that the expected penalty was too low to change predicted behavior relative to the stage 1 baseline. That the low penalty induced more conservative harvest choices indicates that subjects responded to the regulatory frame, not the expected penalty. Velez, et al. (2010) suggest that institutions that rely on framing effects to induce change are more likely to be sensitive to regional differences, whereas those that rely on stronger monetary incentives will yield more consistent decisions. Since the subjects in our study overwhelmingly voted for the imposition of the low penalty, it is not surprising that we observe significant regional differences in individual harvest decisions in the Vote treatments.

Voting effects: The literature on voting for institutional designs argues that this mechanism can coordinate expectations and legitimize regulations via participation. Hence the voting mechanism might increase compliance (decrease harvests) compared to imposed regulations. However, whether voting will have an impact on individual harvest decisions is unclear when an inefficient penalty is chosen, as happened most of the time in our experiments. From Table 7, we generally do not observe significant differences in harvests between treatments in which subjects voted to implement the low penalty and when this low penalty was exogenously imposed. The main exception is in the Caribbean when subjects could communicate. Interestingly, average harvests were significantly higher in this region when 
subjects voted to implement the low penalty than when this penalty was imposed on them. In the Magdalena region, individual harvests were significantly lower when the subjects could communicate and they voted to implement the low penalty in the first five periods of the second stage, but this significance disappeared in the second five periods.

We can also evaluate whether regulatory participation through voting on enforcement levels was more effective at reducing harvests than the exogenously imposed medium penalty, even as subjects usually voted to implement the low penalty. The results in Table 7 suggest that this is not the case. For all three regions, there is usually no difference in harvest choices between the Imposed Medium and Vote(Low) treatments; this result holds regardless of whether subjects could communicate. When there are significant differences- that is, in the Magdalena region in the first five periods when the subjects could not communicate and in the last five periods when they could communicate-individual harvests are lower under the imposed medium penalty than when the subjects voted to implement the low penalty. Our overall conclusion, then, is that voting on the level of enforcement does not reduce individual harvests vis-à-vis exogenously imposed enforcement.

Communication effects when voting: There is a robust literature which suggests that communication in social dilemmas usually results in more efficient decisions (Shankar and Pavitt, 2002, Cardenas et al., 2003, Ostrom, 2010). Contrary to the literature, the results in Table 7 indicate that allowing subjects to communicate before voting did not reduce harvests (relative to voting without prior communication). The one exception is in the Magdalena region; there, communication was effective at reducing harvests during the first five periods of stage 2, but this effect diminished in the later periods. During the experiments, we observed that individuals spent a great deal of their time discussing how to vote, instead of trying to coordinate harvest 
reductions. It appears that the conventional result that communication leads to more efficient choices did not occur in our experiments because the voting decision became the focal point of discussions, which usually resulted in votes to implement the low penalty.

\section{Conclusion}

We have examined, both theoretically and with framed field experiments, individual harvesters' preferences for government enforcement of a harvest quota imposed on the exploitation of a common pool resource. Our theoretical results are unequivocal. In a situation involving poor enforcement of an otherwise efficient harvest quota, individual harvesters will always prefer stricter enforcement. Our experimental results are equally unequivocal in their contradiction of the theoretical result—subjects rarely voted to implement a higher penalty for violating a harvest quota. Consequently, allowing subjects to vote on the design of regulatory enforcement did not lead to more efficient choices than simply imposing a weak enforcement strategy. Others have found similar results in laboratory experiments, but we demonstrate that these results also hold in the field with actual common pool users who operate under poorly enforced fishing regulations. Thus, the reluctance of individuals to vote for stronger enforcement of rules that could make them better off are not likely to be mere artifacts of university labs.

However, recall that other studies of participation in policy design have produced more positive results, so the strong negative reaction to stricter enforcement is a puzzle that could use further investigation. Could it be a general mistrust of the coercive powers of government? Is it due to individuals' inability to discern how increasing the expected costs of their actions could lead to more efficient group behavior? These and other possible explanations for the reluctance 
of individuals and groups to impose efficient enforcement on their behavior can be tested with well-designed experiments.

Studies that have found positive effects of participation in rule design have all been laboratory experiments. We think it is important to conduct these and related experiments in field settings. We have shown that subject behavior is similar in laboratory and field settings in terms of expressing preferences for stricter enforcement, but we do not know if this similarity extends to other dimensions of policy.

The literature, including our contribution, suggests that individual participation is helpful for some dimensions of policy, and not at all helpful for other dimensions. Thus, the value of direct democratic participation in policy design is ambiguous. Clearly, further research is necessary to clarify the benefits and costs of participation and to uncover the most efficient form of participation in the design of policies to confront social dilemmas. 


\section{References}

Alm, J., G.H. McClelland, and W.D. Schulze (1999). "Changing the social norm of tax compliance by voting.” Kyklos 52 (2), 141-71.

Bischoff, I. (2007). "Institutional choice versus communication in social dilemmas - an experimental approach.” Journal of Economic Behavior \& Organization 62(1), 20-36.

Cardenas, J. C., J.K. Stranlund, and C. Willis (2000). "Local Environmental Control and Institutional Crowding-Out." World Development. 28:1719-1733.

Cardenas, J. C. (2005). "Groups, commons and regulations: experiments with villagers and students in Colombia.” In Psychology, Rationality and Economic Behavior: Challenging Standard Assumptions. Bina Agarwal and Alessandro Vercelli (Eds.). Palgrave Macmillan.

Cardenas, J. C., T.K. Ahn and E. Ostrom. (2003). “Communication and cooperation in a common pool resource dilemma: a field experiment.” In Advances in Understanding Strategic Behavior: Game Theory, Experiments and Bounded Rationality: Essays in Honor of Werner Guth. Steffen Huck (Ed.), Palgrave, New York.

Cornes, R., and T. Sandler (1983). “On commons and tragedies.” American Economic Review 73(4), 787-792.

Dolsak, N., and E. Ostrom (2003). “The challenges of the commons.” In The Commons in the New Millennium. N. Dolsak and E. Ostrom editors. Cambridge: MIT Press.

Feld, L.P., and J. R. Tyran. (2002). Tax evasion and voting: an experimental analysis.” Kyklos 55(2), 197-222.

Harrison, G. W. and J. List. “Field experiments.” Journal of Economic Literature 42(4), 2004, 1009-1055.

Margreiter, M., M. Sutter, and D. Dittrich. (2005). "Individual and collective choice and voting in common pool resource problem with heterogeneous actors.” Environmental and Resource Economics 32(2), 241-271.

Ostrom, E., J. Walker and R. Gardner (1994). Rules, Games, and Common-Pool Resources. University of Michigan Press, Ann Arbor Michigan.

Ostrom, E. (2006). The value-added of laboratory experiments for the study of institutions and common-pool resources.” Journal of Economic Behavior and Organization 61(2),149-163.

Ostrom, E. (2010). "Beyond markets and states: polycentric governance of complex economic systems.” American Economic Review 100(3), 641-672.

Shankar, A. and C. Pavitt (2002). "Resource and public goods dilemmas: a new issue for communication research.” Review of Communication 2(3), 251-272. 
Sutter, M., S. Haigner and M. G. Kocher (2010). "Choosing the carrot or the stick? Endogenous institutional choice in social dilemma situations.” Review of Economic Studies 77(4), 15401566.

Tyran, J. R, and L. P. Feld (2006). “Achieving compliance when legal sanctions are nondeterrent.” Scandinavian Journal of Economics 108(1), 135-156.

Vyrastekova, J. and D. van Soest (2003). "Centralized common-pool management and local community participation.” Land Economics 79(4), 500-514.

Velez, M. A., J. K. Stranlund and J. J. Murphy (2009). "What motivates common pool resource users? Experimental evidence from the field.” Journal of Economic Behavior and Organization 70(3), 485-497

Velez, M. A., J. J. Murphy, and J. K. Stranlund (2010). “Centralized and decentralized management of local common pool resources in the developing world: experimental evidence from fishing communities in Colombia.” Economic Inquiry 48(2), 254-265.

Walker, J. M., R. Gardner, A. Herr and E. Ostrom (2000). “Collective choice in the commons: experimental results on proposed allocation rules and votes.” Economic Journal 110(460), 212-34. 
Table 1: Summary statistics of subject characteristics ${ }^{\text {a }}$

\begin{tabular}{|c|c|c|c|c|c|c|}
\hline Subject Characteristics & $\mathrm{N}$ & Caribbean & $\mathrm{N}$ & Magdalena & $\mathrm{N}$ & Pacific \\
\hline Mean Age (years) & 119 & 34 & 120 & 42 & 117 & 40 \\
\hline Mean years of formal education & 118 & 6.4 & 120 & 4.6 & 112 & 5.6 \\
\hline Percent Female & 120 & $53 \%$ & 120 & $15 \%$ & 120 & $13 \%$ \\
\hline $\begin{array}{l}\text { Percent who have lived in the same community } \\
\text { for at least } 10 \text { years. }\end{array}$ & 119 & $80 \%$ & 119 & $92 \%$ & 117 & $93 \%$ \\
\hline Percent for whom fishing is a primary activity & 120 & $65 \%$ & 119 & $89 \%$ & 118 & $91 \%$ \\
\hline
\end{tabular}

${ }^{a} N$ refers to the number of responses. There were 120 participants in each of the three regions. 
Table 2. Experimental Design (Stage 2 treatments)

\begin{tabular}{ccc}
\hline Enforcement & \multicolumn{2}{c}{ Verbal Communication } \\
\cline { 2 - 3 } & Yes & No \\
\hline Low & Imposed Low/Com & Imposed Low/NoCom \\
Medium & Imposed Medium/Com & Imposed Medium/NoCom \\
Vote & Vote(Low or Medium)/Com ${ }^{\mathrm{a}}$ & Vote(Low or Medium)/NoCom ${ }^{\mathrm{a}}$ \\
\hline
\end{tabular}

${ }^{a}$ Voting outcome displayed in parenthesis (Low or Medium) 
Table 3. Individual voting decisions

\begin{tabular}{|c|c|c|c|c|c|c|c|c|c|}
\hline & \multicolumn{3}{|c|}{ Caribbean } & \multicolumn{3}{|c|}{ Magdalena } & \multicolumn{3}{|c|}{ Pacific } \\
\hline & & Low & Medium & & Low & Medium & & Low & Medium \\
\hline & $N$ & Penalty & Penalty & $N$ & Penalty & Penalty & $N$ & Penalty & Penalty \\
\hline No Communication & 200 & 185 & 15 & 200 & 159 & 41 & 200 & 137 & 63 \\
\hline With Communication & 200 & 175 & 25 & 200 & 171 & 29 & 200 & 139 & 61 \\
\hline
\end{tabular}

In each region, there were 4 groups $\times 5$ subjects/group $\times 10$ rounds per group for a total of $N=200$.

Table 4. Group-level voting outcomes

\begin{tabular}{|c|c|c|c|c|c|c|c|c|c|}
\hline & \multicolumn{3}{|c|}{ Caribbean } & \multicolumn{3}{|c|}{ Magdalena } & \multicolumn{3}{|c|}{ Pacific } \\
\hline & & Low & Medium & & Low & Medium & & Low & Medium \\
\hline & $N$ & Penalty & Penalty & $N$ & Penalty & Penalty & $N$ & Penalty & Penalty \\
\hline No Communication & 40 & 40 & 0 & 40 & 36 & 4 & 40 & 35 & 5 \\
\hline With Communication & 40 & 36 & 4 & 40 & 36 & 4 & 40 & 29 & 11 \\
\hline
\end{tabular}

In each region, there were 4 groups $\times 10$ rounds per group for a total of $N=40$. 
Table 5. Mean individual harvest decisions

\begin{tabular}{|c|c|c|c|c|}
\hline \multirow[b]{2}{*}{ Caribbean } & \multicolumn{2}{|c|}{$\begin{array}{c}\text { No } \\
\text { Communication } \\
\end{array}$} & \multicolumn{2}{|c|}{$\begin{array}{c}\text { With } \\
\text { Communication } \\
\end{array}$} \\
\hline & $N$ & Harvest $^{\mathrm{a}}$ & $N$ & Harvest \\
\hline Stage 1 Baseline & 1200 & $\begin{array}{c}5.64 \\
(2.45)\end{array}$ & & \\
\hline Imposed Low & 200 & $\begin{array}{c}5.12 \\
(2.73)\end{array}$ & 200 & $\begin{array}{c}2.91 \\
(2.28)\end{array}$ \\
\hline Imposed Medium & 200 & $\begin{array}{c}4.67 \\
(2.89)\end{array}$ & 200 & $\begin{array}{c}4.73 \\
(2.81)\end{array}$ \\
\hline Vote(Low) $)^{\mathrm{b}}$ & 200 & $\begin{array}{c}4.93 \\
(3.00)\end{array}$ & 180 & $\begin{array}{c}4.42 \\
(1.95)\end{array}$ \\
\hline Vote(Medium) & 0 & 0 & 20 & $\begin{array}{c}4.40 \\
(0.75)\end{array}$ \\
\hline \multicolumn{5}{|l|}{ Magdalena } \\
\hline Stage 1 Baseline & 1200 & $\begin{array}{c}5.55 \\
(2.51)\end{array}$ & & \\
\hline Imposed Low & 200 & $\begin{array}{c}4.35 \\
(2.76)\end{array}$ & 200 & $\begin{array}{c}4.76 \\
(2.63)\end{array}$ \\
\hline Imposed Medium & 200 & $\begin{array}{l}3.85 \\
(2.66)\end{array}$ & 200 & $\begin{array}{c}3.13 \\
(2.36)\end{array}$ \\
\hline Vote(Low) & 180 & $\begin{array}{c}4.89 \\
(2.76)\end{array}$ & 180 & $\begin{array}{c}3.81 \\
(2.39)\end{array}$ \\
\hline Vote(Medium) & 20 & $\begin{array}{l}2.40 \\
(1.64)\end{array}$ & 20 & $\begin{array}{c}2.65 \\
(1.79)\end{array}$ \\
\hline \multicolumn{5}{|l|}{ Pacific } \\
\hline Stage 1 Baseline & 1200 & $\begin{array}{c}5.63 \\
(2.37)\end{array}$ & & \\
\hline Imposed Low & 200 & $\begin{array}{l}3.26 \\
(2.11)\end{array}$ & 200 & $\begin{array}{c}2.96 \\
(1.96)\end{array}$ \\
\hline Imposed Medium & 200 & $\begin{array}{c}3.94 \\
(2.42)\end{array}$ & 200 & $\begin{array}{c}3.41 \\
(2.26)\end{array}$ \\
\hline Vote(Low) & 175 & $\begin{array}{c}3.26 \\
(2.09)\end{array}$ & 145 & $\begin{array}{c}3.31 \\
(2.38)\end{array}$ \\
\hline Vote(Medium) & 25 & $\begin{array}{c}4.04 \\
(2.72)\end{array}$ & 55 & $\begin{array}{c}5.29 \\
(2.68)\end{array}$ \\
\hline
\end{tabular}

\footnotetext{
${ }^{a}$ Standard deviation in parentheses.

${ }^{\mathrm{b}}$ Text in parentheses following the Vote treatment label refers to the outcome.
} 
Table 6: Random effects Tobit estimation of individual harvest decisions in stage $2^{\mathrm{a}}$

\begin{tabular}{|c|c|c|c|c|}
\hline \multirow{2}{*}{$\begin{array}{l}\text { Variable } \\
\text { Age (years) }\end{array}$} & \multicolumn{2}{|c|}{ First 5 Periods } & \multicolumn{2}{|c|}{ Last 5 Periods } \\
\hline & $-0.02 * *$ & $(0.01)$ & $-0.03 * * *$ & $(0.01)$ \\
\hline Education (years of formal schooling) & $0.11^{* * *}$ & $(0.04)$ & $0.13^{* * *}$ & $(0.05)$ \\
\hline Female & -0.12 & $(0.31)$ & 0.29 & $(0.36)$ \\
\hline Lived in region for at least 10 years & 0.23 & $(0.39)$ & -0.02 & $(0.45)$ \\
\hline Fisherman & $0.75 * *$ & $(0.34)$ & $1.23 * * *$ & $(0.39)$ \\
\hline Period & $0.08 * *$ & $(0.04)$ & $0.09 * *$ & $(0.04)$ \\
\hline \multicolumn{5}{|l|}{ Caribbean Region (Car) } \\
\hline Car $\times$ Imposed Low / NoCom & $3.19 * * *$ & $(0.95)$ & $2.86 * *$ & $(1.19)$ \\
\hline Car × Imposed Low / Com & 1.25 & $(0.95)$ & -0.69 & (1.19) \\
\hline Car $\times$ Imposed Med / NoCom & $3.12 * * *$ & $(0.94)$ & $2.71 * *$ & $(1.17)$ \\
\hline Car × Imposed Med / Com & $3.56 * * *$ & $(0.97)$ & $2.10 *$ & $(1.20)$ \\
\hline Car $\times$ Vote $($ Low $) /$ NoCom & $2.98 * * *$ & $(0.99)$ & $2.76^{* *}$ & $(1.23)$ \\
\hline Car $\times$ Vote $($ Low $) /$ Com & $2.98 * * *$ & $(0.93)$ & $2.17 *$ & $(1.16)$ \\
\hline \multicolumn{5}{|l|}{ Magdalena Region (Mag) } \\
\hline Mag $\times$ Imposed Low / NoCom & $2.88 * * *$ & $(0.96)$ & $3.01 * *$ & $(1.20)$ \\
\hline Mag $\times$ Imposed Low / Com & $2.76 * * *$ & $(0.96)$ & $3.30 * * *$ & $(1.20)$ \\
\hline Mag $\times$ Imposed Med / NoCom & $1.62 *$ & $(0.93)$ & $1.96 *$ & $(1.16)$ \\
\hline Mag $\times$ Imposed Med / Com & 1.28 & $(0.96)$ & 0.84 & $(1.20)$ \\
\hline Mag $\times$ Vote $($ Low $) /$ NoCom & $3.59 * * *$ & $(0.98)$ & $3.07 * *$ & (1.19) \\
\hline Mag $\times$ Vote $($ Low $) /$ Com & 1.22 & $(0.93)$ & $2.13^{*}$ & $(1.16)$ \\
\hline \multicolumn{5}{|l|}{ Pacific Region (Pac) } \\
\hline Pac $\times$ Imposed Low / NoCom & 1.46 & $(0.97)$ & 0.96 & $(1.20)$ \\
\hline Pac $\times$ Imposed Low / Com & 0.67 & $(1.02)$ & -0.20 & $(1.26)$ \\
\hline Pac $\times$ Imposed Med / NoCom & $2.22 * *$ & $(0.96)$ & $2.13 *$ & $(1.20)$ \\
\hline Pac $\times$ Imposed Med / Com & $1.85 *$ & $(0.96)$ & 1.09 & $(1.20)$ \\
\hline Pac $\times$ Vote $($ Low $) /$ NoCom & 1.35 & $(0.99)$ & 0.97 & $(1.23)$ \\
\hline Pac $\times$ Vote $($ Low $) /$ Com & $1.62 *$ & $(0.96)$ & -0.20 & $(1.27)$ \\
\hline Number of observations & \multicolumn{2}{|c|}{1591} & \multicolumn{2}{|c|}{1582} \\
\hline Prob $>\chi^{2}$ & \multicolumn{2}{|c|}{0.00} & \multicolumn{2}{|c|}{0.00} \\
\hline
\end{tabular}

a The dependent variable is the individual's harvest, $x_{i} \in[1,9]$.

${ }^{\mathrm{b}}$ Since the data only includes stage two, period ranges from 11 to 20 .

*** denotes $p \leq 0.01$; ** denotes $p \leq 0.05$; * denotes $p \leq 0.10$. 
Table 7: Wald tests of hypotheses regarding individual harvest decisions based on the model reported in Table 6

\begin{tabular}{|c|c|c|c|}
\hline \multicolumn{2}{|l|}{ Test } & $\begin{array}{l}\text { First } 5 \\
\text { Periods }\end{array}$ & $\begin{array}{c}\text { Last } 5 \\
\text { Periods }\end{array}$ \\
\hline \multicolumn{4}{|l|}{ Regional differences } \\
\hline $\begin{array}{l}H_{0}: \text { Caribbean }=\text { Magdalena }= \\
\text { Imposed Low/NoCom } \\
\text { Imposed Low/Com } \\
\text { Imposed Medium/NoCom } \\
\text { Imposed Medium/Com } \\
\text { Vote (Low)/NoCom } \\
\text { Vote (Low)/Com }\end{array}$ & Pacific for a given treatm & $\begin{array}{l}0.02^{* *} \\
0.01 * * * \\
0.10^{*} \\
0.00^{* * *} \\
0.00^{* * *} \\
0.04^{* *}\end{array}$ & $\begin{array}{l}0.01 * * * \\
0.00 * * * \\
0.64 \\
0.22 \\
0.02 * * \\
0.01 * * *\end{array}$ \\
\hline \multicolumn{4}{|l|}{ Voting effects, by region } \\
\hline $\begin{array}{l}\mathrm{H}_{0}: \text { Imposed Low/NoCom } \\
\mathrm{H}_{0}: \text { Imposed Low/Com } \\
\mathrm{H}_{0}: \text { Imposed Medium/NoCom } \\
\mathrm{H}_{0}: \text { Imposed Medium/Com }\end{array}$ & $\begin{array}{l}=\text { Vote }(\text { Low }) / \text { NoCom } \\
=\text { Vote }(\text { Low }) / \text { Com } \\
=\text { Vote }(\text { Low }) / \text { NoCom } \\
=\text { Vote }(\text { Low }) / \text { Com }\end{array}$ & $\begin{array}{l}0.76 \\
0.02 * * \\
0.84 \\
0.41\end{array}$ & $\begin{array}{l}0.90 \\
0.00 * * * \\
0.95 \\
0.93\end{array}$ \\
\hline $\begin{array}{l}\text { Magdalena } \\
\mathrm{H}_{0} \text { : Imposed Low/NoCom } \\
\mathrm{H}_{0} \text { : Imposed Low/Com } \\
\mathrm{H}_{0} \text { : Imposed Medium /NoCom } \\
\mathrm{H}_{0} \text { : Imposed Medium /Com }\end{array}$ & $\begin{array}{l}=\text { Vote }(\text { Low }) / \text { NoCom } \\
=\text { Vote }(\text { Low }) / \text { Com } \\
=\text { Vote }(\text { Low }) / \text { NoCom } \\
=\text { Vote }(\text { Low }) / C o m\end{array}$ & $\begin{array}{l}0.30 \\
0.02 * * * \\
0.00^{* * *} \\
0.93\end{array}$ & $\begin{array}{l}0.94 \\
0.12 \\
0.15 \\
0.08^{*}\end{array}$ \\
\hline $\begin{array}{l}\text { Pacific } \\
\mathrm{H}_{0} \text { : Imposed Low/NoCom } \\
\mathrm{H}_{0} \text { : Imposed Low/Com } \\
\mathrm{H}_{0} \text { : Imposed Medium /NoCom } \\
\mathrm{H}_{0} \text { : Imposed Medium /Com }\end{array}$ & $\begin{array}{l}=\text { Vote }(\text { Low }) / \text { NoCom } \\
=\text { Vote }(\text { Low }) / \text { Com } \\
=\text { Vote }(\text { Low }) / \text { NoCom } \\
=\text { Vote }(\text { Low }) / \text { Com }\end{array}$ & $\begin{array}{l}0.87 \\
0.18 \\
0.20 \\
0.75\end{array}$ & $\begin{array}{l}0.99 \\
1.00 \\
0.15 \\
0.15\end{array}$ \\
\hline $\begin{array}{l}\text { Communication effects when votir } \\
H_{0}: \text { Vote }(\text { Low }) / \text { NoCom }=\text { Vote } \\
\text { Caribbean } \\
\text { Magdalena } \\
\text { Pacific }\end{array}$ & $\begin{array}{l}\text { 1g, by region } \\
\text { Low)/Com for a given }\end{array}$ & $\begin{array}{l}0.99 \\
0.00 * * * \\
0.69\end{array}$ & $\begin{array}{l}0.46 \\
0.21 \\
0.19 \\
\end{array}$ \\
\hline
\end{tabular}

$* * *$ denotes $p \leq 0.01$; ** denotes $p \leq 0.05$; * denotes $p \leq 0.10$. 


\section{Reviewer's Appendix: Experiment Instructions}

Before we begin, we want to thank you all for accepting this invitation and participating in this exercise. The objective of this exercise is to understand how people make decisions related to the use of a shared natural resource. All the decisions you make, as well as all the other information you will provide us, will remain confidential. We will not divulge your individual decisions to any other member of the community, nor to any other person.

\section{Introduction}

The exercise in which you are going to participate can be different from other exercises in which members of your community might have participated in the past, therefore, any comment that you might have heard about the exercise does not necessarily apply to the version in which you will participate.

This exercise is similar to a situation in which a group of people have to make decisions on how to use a shared natural resource. For example, a forest, a drinking water source, or a fishing area. In this experiment, the resource will be referred as a fishery.

You have been selected to participate in a group of 5 people. Today, there are 3 groups participating at the same time. However, each group is independent and the decisions of the other groups do not affect the decisions of your group. Each group will be differentiated by the color of the sheets used during the exercise.

In this exercise you will earn money depending on your decisions and the decisions of the other members of your group. The reason why we use money in this exercise is to represent real life situations in which your economic decisions will bring yourself monetary consequences. You will play several rounds equivalent, for example, to periods such as years, months, or fishing seasons.

In each round, you will earn a number of points that will be equivalent to a number of pesos. At the end of the exercise, we will sum the total number of pesos earned in all the rounds, we will round the total earned, and we will personally hand that to you in cash.

We will now explain how to participate in the exercise. Please pay a lot of attention to the instructions. If you understand the instructions, you will be able to make better decisions in the exercise. Please, remain seated and do not speak with other participants. If you have a question, raise you hand. The assistant will answer your question in private.

\section{Earnings Table}

We will now hand out the EARNINGS TABLE which contains all the information you will need to make your decisions in this exercise.

All participants have the same EARNINGS TABLE that you do. The numbers in the table are points equivalent to the pesos you can earn in each round, depending on both what you decide to extract and the decisions made by others in your group. 
In each round you have to decide how many units of the resource you will extract. We will call your decision "MY LEVEL OF EXTRACTION.” These units correspond to the columns 1 to 9 in the EARNING TABLE. In this exercise, each participant can extract a maximum of 9 units, and a minimum of 1 .

In the EARNINGS TABLE, the decisions of the other members of your group correspond to the column "LEVEL OF EXTRACTION OF OTHERS", which will be a number between 4 and 36. This number is the sum of the units extracted by the other members of the group. In other words, "LEVEL OF EXTRACTION OF OTHERS" is equal to: the total extraction of the whole group, minus the amount you extracted. When you make your decision, you will not know the decisions made by the other members of your group.

Once all participants hand in their decisions, we will sum all the levels of extraction and will announce the group's TOTAL LEVEL OF EXTRACTION. With this information you will be able to calculate the "LEVEL OF EXTRACTION OF OTHERS," which is equal to the "TOTAL LEVEL OF EXTRACTION” minus "MY LEVEL OF EXTRACTION”.

Let's see some examples so that you can understand how to use the EARNINGS TABLE.

Imagine you decide that "MY LEVEL OF EXTRACTION" is 4 units, and that the other members of the group extract 4 units each. We will announce that the TOTAL level of extraction is 20 units. Since you decided to extract 4, you can calculate the "LEVEL OF EXTRACTION OF OTHERS," which is equal to the "TOTAL LEVEL OF EXTRACTION" minus "MY LEVEL OF EXTRACTION". In this case, the "LEVEL OF EXTRACTION OF OTHERS" is $20-4=16$ units. Thus, as seen in the table, your earnings will be 859 .

In the previous example all the members of the group picked the same level of extraction. However, each person can pick a different number. For example, if you choose 4 and the other members of the group extract 2, 3, 7 and 8, we will announce that the TOTAL level of extraction is 24. Given the fact that you decided to extract 4, the "LEVEL OF EXTRACTION OF OTHERS" will be 20. In other words, the "TOTAL LEVEL OF EXTRACTION (24) minus "MY LEVEL OF EXTRACTION" (4). In this case, as seen on the table, your earnings will be 754.

The EARNINGS TABLE has an additional table called "Average of others". This column indicates you the average decision of your group for a determined level. For example, if the others extract 8 , this means that the average amount extracted per person is 2 . Instead, if the others extract 20, the average amount extracted per person is 5 .

Take a few seconds to look at the EARNINGS TABLE and understand how it works. If you have any questions, please raise your hand and someone will come to you. 


\section{Decision Card}

I will now explain how you will inform us in each round your level of extraction. In each round you will receive a “decision card”. The decision cards are these small pieces of paper.

\begin{tabular}{|c|c|}
\hline \multicolumn{2}{|c|}{ DECISION CARD } \\
\hline Participant Number: & \\
\hline Round Number: & \\
\hline $\begin{array}{c}\text { My level of extrac1tion: } \\
\text { (a number between 1 }-9 \text { ): }\end{array}$ & \\
\hline $\begin{array}{c}\text { How much do you think } \\
\text { others will extract? } \\
\text { (a number between 4 -36): }\end{array}$ & \\
\hline
\end{tabular}

In each round you will have to write:

- The number of the round, which will be announced by us.

-“MY LEVEL OF EXTRACTION”, in other words, how many units will you extract, which in this case will be a number between 1 and 9 .

-You also have to write what you think the other members of your group will extract.

This is the sum of the levels of extraction that you think the other 4 members of your group will extract. This sum is a number between 4 and 36. Remember that when you make your decision you do not know what the others are choosing. However, we want to know how much you think the others will extract. For example, if you think that two people will choose 3 and the other two 5 , then, what you think the others will extract is $16(3+3+5+5)$.

What you write on the level of extraction of others will not affect your earnings, either if it is equal or different to what actually happened. However, we are interested to know what you are thinking about the level of extraction of the others when you make your choice. 
After all the members of your group have made their decisions, we will pick up the 5 participants' cards and calculate the groups' TOTAL level of extraction. Once we announce the total extraction of the group you will be able to calculate the true "LEVEL OF EXTRACTION

OF OTHERS.” With this information and your level of extraction, you will be able to calculate how much you earned by looking at the EARNINGS TABLE.

It is very important that you remember that your decisions are private and that you can not show them to the other members of the group. We will only announce the TOTAL level of extraction.

\section{Calculation sheet}

Each one of you will receive a calculation sheet with which you record your decisions and earnings. Please write your participant number in the calculation sheet. This is the same number that is written in the decision cards.

Let's see how to use the calculation sheet by looking at an example. Suppose you decided to extract 4 units. In consequence, you have to write 4 under column A of the calculation sheet, as shown in the example. You should also write this number in "MY LEVEL OF EXTRACTION" in the Decision Card .You are writing your decision in two places, in the Decision Card, which you will hand in back to us, and in the calculations sheet. Please, check that you have written the same number in the two sheets before you hand in the decision card.

After all the members of the group have finished taking their decisions, we will pick up the cards of the 5 participants and calculate the groups' TOTAL level of extraction.

Suppose the "TOTAL LEVEL OF EXTRACTION" is 20 units. You should write 20 in the column B in the calculations sheet. In order to calculate accurately the "LEVEL OF EXTRACTION OF OTHERS," you should subtract Column A ("MY LEVEL OF EXTRACTION”) from Column B (“TOTAL LEVEL OF EXTRACTION”) You should write the result in Column $C$ ("LEVEL OF EXTRACTION OF OTHERS") In our example, the “LEVEL OF EXTRACTION OF OTHERS” is $16(20-4$.

In order to calculate your earnings, you should use the EARNINGS TABLE. In this case, given that "MY LEVEL OF EXTRACTION" is 4 and the "LEVEL OF EXTRACTION OF OTHERS" is 16 , then your earnings will be 859 . This is the information you should write in column D.

\section{Practice rounds}

Before we begin the exercise we will do some practice rounds. The decisions that you take in these practice rounds would not affect your earnings today.

The first practice round will be done altogether. First, write the number of the round in the decision card, in this case (P) of practice. After that, looking at the EARNINGS TABLE suppose that each one of you picked 5. Write this in the decision card and in Column A of the earnings sheet. You should also write in the decision card what you think the other members of your 
group will extract. In this case, it is 20, because we know that all of them picked 5. Remember, when we begin the real exercise, you will not know the exact number of extraction of the other members while you will be picking your level of extraction. In the next rounds you will write what you think the others will extract.

Given that all the members of the group picked 5 in this example, the total level of extraction for the group is 25. Each one should write now 25 under Column B ("TOTAL LEVEL OF EXTRACTION") in the calculations sheet.

Now subtract "MY LEVEL OF EXTRACTION" (5) from the "TOTAL LEVEL OF EXTRACTION” (25). In other words, column B minus Column A. This operation is equal to 20. This number is the true "LEVEL OF EXTRACTION OF OTHERS", which you should write in Column C. Using the number in Column A, "MY LEVEL OF EXTRACTION," and the number under column C, the "LEVEL OF EXTRACTION OF OTHERS", you should use the earnings table to determine your earnings for this round. In this case, your earnings will be 790. Write your earnings in column D.

We did this example and the previous one supposing that everyone picked the same level of extraction. However, when you make your decision, you may choose the level of extraction that you want by looking at the EARNINGS TABLE. Are there any questions?

Let's continue with the next practice round. First, write down the round's name in the decision card, in this case (P) of practice. Now, each one of you has to decide your level of extraction using the EARNINGS TABLE. Write it down in the decision card and in Column $\mathrm{A}$ in the calculations sheet. Before you hand in the decision card, check that the number in column A is equal to the one you wrote in "MY LEVEL OF EXTRACTION" in the decision card. You should also write in the decision card the level of extraction that you believe the other members of the group will extract. 
EARNINGS TABLE

\begin{tabular}{|c|c|c|c|c|c|c|c|c|c|c|}
\hline \multirow[b]{2}{*}{$\begin{array}{l}\text { Level of } \\
\text { extraction } \\
\text { of others }\end{array}$} & \multicolumn{9}{|c|}{ My level of extraction } & \multirow[b]{2}{*}{$\begin{array}{c}\text { Average } \\
\text { of the } \\
\text { others }\end{array}$} \\
\hline & 1 & 2 & 3 & 4 & 5 & 6 & 7 & 8 & 9 & \\
\hline 4 & 900 & 996 & 1087 & 1172 & 1252 & 1326 & 1395 & 1458 & 1516 & 1.0 \\
\hline 5 & 882 & 976 & 1064 & 1146 & 1223 & 1295 & 1361 & 1421 & 1476 & 1.3 \\
\hline 6 & 864 & 955 & 1040 & 1120 & 1194 & 1263 & 1326 & 1384 & 1436 & 1.5 \\
\hline 7 & 846 & 934 & 1017 & 1094 & 1165 & 1231 & 1292 & 1347 & 1396 & 1.8 \\
\hline 8 & 829 & 914 & 994 & 1068 & 1137 & 1200 & 1258 & 1310 & 1357 & 2.0 \\
\hline 9 & 811 & 893 & 970 & 1042 & 1108 & 1168 & 1223 & 1273 & 1317 & 2.3 \\
\hline 10 & 793 & 873 & 947 & 1016 & 1079 & 1137 & 1189 & 1236 & 1277 & 2.5 \\
\hline 11 & 775 & 852 & 923 & 989 & 1050 & 1105 & 1154 & 1198 & 1237 & 2.8 \\
\hline 12 & 757 & 831 & 900 & 963 & 1021 & 1073 & 1120 & 1161 & 1197 & 3.0 \\
\hline 13 & 739 & 811 & 877 & 937 & 992 & 1042 & 1086 & 1124 & 1157 & 3.3 \\
\hline 14 & 721 & 790 & 853 & 911 & 963 & 1010 & 1051 & 1087 & 1117 & 3.5 \\
\hline 15 & 703 & 769 & 830 & 885 & 934 & 978 & 1017 & 1050 & 1077 & 3.8 \\
\hline 16 & 686 & 749 & 807 & 859 & 906 & 947 & 983 & 1013 & 1038 & 4.0 \\
\hline 17 & 668 & 728 & 783 & 833 & 877 & 915 & 948 & 976 & 998 & 4.3 \\
\hline 18 & 650 & 708 & 760 & 807 & 848 & 884 & 914 & 939 & 958 & 4.5 \\
\hline 19 & 632 & 687 & 736 & 780 & 819 & 852 & 879 & 901 & 918 & 4.8 \\
\hline 20 & 614 & 666 & 713 & 754 & 790 & 820 & 845 & 864 & 878 & 5.0 \\
\hline 21 & 596 & 646 & 690 & 728 & 761 & 789 & 811 & 827 & 838 & 5.3 \\
\hline 22 & 578 & 625 & 666 & 702 & 732 & 757 & 776 & 790 & 798 & 5.5 \\
\hline 23 & 560 & 604 & 643 & 676 & 703 & 725 & 742 & 753 & 758 & 5.8 \\
\hline 24 & 543 & 584 & 620 & 650 & 675 & 694 & 708 & 716 & 719 & 6.0 \\
\hline 25 & 525 & 563 & 596 & 624 & 646 & 662 & 673 & 679 & 679 & 6.3 \\
\hline 26 & 507 & 543 & 573 & 598 & 617 & 631 & 639 & 642 & 639 & 6.5 \\
\hline 27 & 489 & 522 & 549 & 571 & 588 & 599 & 604 & 604 & 599 & 6.8 \\
\hline 28 & 471 & 501 & 526 & 545 & 559 & 567 & 570 & 567 & 559 & 7.0 \\
\hline 29 & 453 & 481 & 503 & 519 & 530 & 536 & 536 & 530 & 519 & 7.3 \\
\hline 30 & 435 & 460 & 479 & 493 & 501 & 504 & 501 & 493 & 479 & 7.5 \\
\hline 31 & 417 & 439 & 456 & 467 & 472 & 472 & 467 & 456 & 439 & 7.8 \\
\hline 32 & 400 & 419 & 433 & 441 & 444 & 441 & 433 & 419 & 400 & 8.0 \\
\hline 33 & 382 & 398 & 409 & 415 & 415 & 409 & 398 & 382 & 360 & 8.3 \\
\hline 34 & 364 & 378 & 386 & 389 & 386 & 378 & 364 & 345 & 320 & 8.5 \\
\hline 35 & 346 & 357 & 362 & 362 & 357 & 346 & 329 & 307 & 280 & 8.8 \\
\hline 36 & 328 & 336 & 339 & 336 & 328 & 314 & 295 & 270 & 240 & 9.0 \\
\hline
\end{tabular}




\section{Stage 2 - Imposed Medium/NoCom}

(These are the instructions for the medium penalty of 165. The instructions for the Imposed low penalty treatment are identical, except that the penalty for each additional unit extracted is 27).

We will now begin the second part of this exercise. This part is very similar to the previous one, with the difference that in the next rounds we will establish a regulation. The objective of this regulation is to make all participants of your group extract $\mathbf{2}$ units.

In order to promote that people do not extract more than 2 units of the resource, we are going to impose a penalty of 165 points for each additional unit extracted. The table that we will be handing out summarizes the total penalties that result from each level of extraction.

(Hand out the tables and explain them)

\section{Penalty Table}

\begin{tabular}{|c|c|c|c|c|c|c|c|c|c|}
\hline $\begin{array}{c}\text { My level of } \\
\text { extraction }\end{array}$ & $\mathbf{1}$ & $\mathbf{2}$ & $\mathbf{3}$ & $\mathbf{4}$ & $\mathbf{5}$ & $\mathbf{6}$ & $\mathbf{7}$ & $\mathbf{8}$ & $\mathbf{9}$ \\
\hline Penalty & 0 & 0 & 165 & 330 & 495 & 660 & 825 & 990 & 1155 \\
\hline
\end{tabular}

You will have to pay the penalty only if you are inspected when extracting more than 2 units of the resource. In other words, you can extract more than 2 units, but if you are inspected, you will have to pay the penalty. However, it will be very difficult to inspect the decisions of all the members of the group.

In consequence, once each person decides their level of extraction (a number between 1 and 9) and has handed in the decision card, we will randomly pick who will be inspected.

In order to decide who will be inspected, we will take a ballot from a bag containing 5 ballots with the participants' numbers on them, and 5 other blank ballots.

(Show the ballots)

This implies that for each round you have ONE chance in 10 of being inspected. If your number is selected and you extracted more than 2 units of the resource, then you will have to pay the penalty for every additional unit. Nobody else will ever know the result of such inspection. If a blank ballot is selected, no one will be inspected. 


\section{(Practice picking the ballot)}

In each round we will only take one ballot. The selected ballot will be returned to the bag. This means that the result of the inspection in this round will not affect the result of the next one. In this way, someone can be inspected more than one time during the exercise. It is also possible that you never get inspected.

\section{Let's make some examples:}

For example, if you extract 5 units of the resource, your level of extraction is 3 units greater than the permitted level of 2 units.

If your number is randomly selected from the bag, then you will be inspected. Since you extracted 5 units, the penalty will be 495, as can be seen in the penalty table $(5-2=3$ and $3 * 165=495$ ). In consequence, we will have to subtract 495 from your earnings.

If a blank ballot is selected nobody will be inspected and we will follow on to the next round. If you are not inspected, you will not have to pay any penalty.

Another example:

If you extract 2 units, your level of extraction is equal to the level permitted. If your number is randomly selected from the bag, you will be inspected. Given the fact that your level of extraction is equal to the permitted level, then you will not have to pay the penalty. If a blank ballot is selected, nobody will be inspected and we will pass to the next round. If you are not inspected, you will not have to pay the penalty.

\section{Calculation sheet}

The calculation sheet for these rounds is very similar to the one used in the previous rounds, but includes some changes.

Before we begin, please write your participant's number in the new calculation sheet.

Columns A, B and C are used as in previous rounds. In column D you must write your earnings before you know if you will be inspected. Now, there are two additional columns. In column E you must write whether you were inspected or not in each round. Write YES or NO. In column $\mathrm{F}$ we will write the total value of the penalty you will have to pay if you are inspected. If you were not inspected, please write 0 in this column. In the last column, column G, you can write your earnings after the inspection. Earnings after the inspection are calculated subtracting the value of the penalty (column F) from the earnings before the inspection (column D). If you were not inspected, or your penalty is 0 , your earnings (column $G$ ) will be exact to what was written in column D (My earnings before the inspection).

Let's see some examples:

Suppose that your level of extraction was 5 units and the total level of extraction was 17 units. Write this information in the corresponding columns as in the previous rounds. In this case, level of extraction of the others is 12 (column C) and your earnings will be 1021, before the inspection.

If your number is randomly selected from the bag, then you will be inspected. Write "YES" in column E. Since you extracted 5 units, the penalty will be 495, as you can see in the penalty table 
(5-2 =3 and 3*165=495.) Write 495 in column F (total penalty value.) In consequence, your total earnings for this round will be 1021 (column D) - 495 (column F) $=526$. Write 526 in the last column (My earnings after the inspection, column G.)

If a blank ballot is selected and nobody is inspected we will pass the following round. You will not have to pay any penalty. Write NO in column E, 0 in column F (penalty value) and write your earnings before the inspection (column $\mathrm{D}$ ) in column $\mathrm{G}$ (earnings after the inspection.)

\section{Stage 2 - Imposed Medium/Com}

(These instructions are identical to the medium penalty treatment explained above, except that after the practice rounds are over, participants are informed that communication is allowed. The instructions for the low penalty and communication treatment are identical, except that the penalty for each additional unit extracted is 27)

At the beginning of each round, before you decide how much you will extract, you will have 5 minutes to discuss the exercise with the other members of your group. During these 5 minutes, you will be able to discuss with them anything related with the exercise. Before the discussion round begins, turn your calculations sheet upside down and leave it on the table. After 5 minutes, the discussion will come to an end and you will return to your seat to decide privately what your level of extraction decision will be.

\section{Stage 2 - Vote(Low or Medium)/NoCom}

We will now begin the second part of this exercise. This part is very similar to the previous one, with the difference that in the next rounds we will help you to establish a regulation. The objective of this regulation is that all participants of your group extract $\mathbf{2}$ units.

The idea is to give you the opportunity to participate in the regulatory design. In order to promote that people do not extract more than 2 units of the resource, your group will choose the penalty for each additional unit extracted that a participant will have to pay if inspected when extracting more than 2 units of the resource.

In each round, before you decide your level of extraction, the group will choose the penalty for each additional unit extracted. To do so, each participant will vote for the preferred penalty. The penalty with the majority of votes will be imposed.

You will have two penalty options as is written in the voting cards:

- a penalty of 27 points for each additional unit extracted

- a penalty of 165 points for each additional unit extracted

At the beginning of each round you will mark with an $\mathrm{X}$ your preferred choice. Please mark only one option and do not return your voting card without marking an option. Your vote will 
be private, that is, nobody will know your choice. In the voting card please also write the round number.

The instructor will collect the votes and announce the results. The penalty with 3 or more votes will be the penalty for that round only. At the beginning of each round the voting will be repeated. When the voting process have finished you will proceed to choose your level of extraction.

The table that we will be handing out summarizes the total penalties that result from each level of extraction for each type of penalty.

\begin{tabular}{|c|c|c|c|c|c|c|c|c|c|}
\hline $\begin{array}{c}\text { My level of } \\
\text { extraction }\end{array}$ & $\mathbf{1}$ & $\mathbf{2}$ & $\mathbf{3}$ & $\mathbf{4}$ & $\mathbf{5}$ & $\mathbf{6}$ & $\mathbf{7}$ & $\mathbf{8}$ & $\mathbf{9}$ \\
\hline $\begin{array}{c}\text { Penalty- } \\
\mathbf{1 6 5}\end{array}$ & 0 & 0 & 165 & 330 & 495 & 660 & 825 & 990 & 1155 \\
\hline
\end{tabular}

\begin{tabular}{|c|c|c|c|c|c|c|c|c|c|}
\hline $\begin{array}{c}\text { My level of } \\
\text { extraction }\end{array}$ & $\mathbf{1}$ & $\mathbf{2}$ & $\mathbf{3}$ & $\mathbf{4}$ & $\mathbf{5}$ & $\mathbf{6}$ & $\mathbf{7}$ & $\mathbf{8}$ & $\mathbf{9}$ \\
\hline Penalty- 27 & 0 & 0 & 27 & 54 & 81 & 108 & 135 & 162 & 189 \\
\hline
\end{tabular}

You will have to pay the penalty only if you are inspected when extracting more than 2 units of the resource. In other words, you can extract more than 2 units, but if you are inspected, you will have to pay the penalty. However, it will be very difficult to inspect the decisions of all the members of the group.

In consequence, once each person decides their level of extraction (a number between 1 and 9) and has handed in the decision card, we will randomly pick who will be inspected.

In order to decide who will be inspected, we will take a ballot from a bag containing 5 ballots with the participants' numbers on them, and 5 other blank ballots.

(Show the ballots)

This implies that for each round you have ONE chance in 10 of being inspected. If your number is selected and you extracted more than 2 units of the resource, then you will have to pay the penalty for every additional unit. Nobody else will ever know the result of such inspection. If a blank ballot is selected, no one will be inspected. 
(Practice picking the ballot)

In each round we will only take one ballot. The selected ballot will be returned to the bag. This means that the result of the inspection in this round will not affect the result of the next one. In this way, someone can be inspected more than one time during the exercise. It is also possible that you never get inspected.

\section{Some examples:}

-Lets suppose that 3 participants voted for the penalty of 165 points and 2 for the penalty of 27 points. Then, because the majority voted for the penalty of 165 that will be the penalty for each additional unit extract in that round.

For example, if you extract 5 units of the resource, your level of extraction is 3 units greater than the permitted level of 2 units.

If your number is randomly selected from the bag, then you will be inspected. Since you extracted 5 units, the penalty will be 495 , as can be seen in the penalty table ( $5-2=3$ and $3 * 165=495)$. In consequence, we will have to subtract 495 from your earnings.

If a blank ballot is selected nobody will be inspected and we will follow on to the next round. If you are not inspected, you will not have to pay any penalty.

Another example:

If you extract 2 units, your level of extraction is equal to the level permitted. If your number is randomly selected from the bag, you will be inspected. Given the fact that your level of extraction is equal to the permitted level, then you will not have to pay the penalty. If a blank ballot is selected, nobody will be inspected and we will pass to the next round. If you are not inspected, you will not have to pay the penalty

\section{Calculation sheet}

The calculation sheet for these rounds is very similar to the one used in the previous rounds, but includes some changes.

Before we begin, please write your participant's number in the new calculation sheet.

Columns A, B and C are used as in previous rounds. In column D you must write your earnings before you know if you will be inspected. Now, there are four additional columns. In column E you must write the penalty chosen by the group (27 or 165). In column F you must write whether you were inspected or not in each round. Write YES or NO. In column G we will write the total value of the penalty you will have to pay if you are inspected. If you were not inspected, please write 0 in this column. In the last column, column $\mathrm{H}$, you can write your earnings after the inspection. Earnings after the inspection are calculated subtracting the value of the penalty (column G) from the earnings before the inspection (column D). If you were not inspected, or your penalty is 0 , your earnings (column $\mathrm{H}$ ) will be exact to what was written in column D (My earnings before the inspection).

Let's see some examples:

Suppose that 3 participants voted for the penalty of 165 points and 2 for the penalty of 27 points. Then, because the majority voted for the penalty of 165 that will be the penalty for each additional unit extract in that round. Please write 165 in column E. 
Suppose that your level of extraction was 5 units and the total level of extraction was 17 units. Write this information in the corresponding columns as in the previous rounds. In this case, level of extraction of the others is 12 (column C) and your earnings will be 1021, before the inspection. Write this information in column D.

If your number is randomly selected from the bag, then you will be inspected. Write "YES" in column F. Since you extracted 5 units, the penalty will be 495, as you can see in the penalty table (5-2 =3 and $3 * 165=495$.) Write 495 in column $G$ (total penalty value.) In consequence, your total earnings for this round will be 1021 (column D) - 495 (column G) = 526. Write 526 in the last column (My earnings after the inspection, column H.)

If a blank ballot is selected and nobody is inspected we will pass the following round. You will not have to pay any penalty. Write NO in column F, 0 in column G (penalty value) and write your earnings before the inspection (column D) in column $\mathrm{H}$ (my earnings after the inspection.)

\section{Stage 2 - Vote(Low or Medium)/Com}

(These instructions are identical to voting treatment explained above, except that after the practice rounds are over, participants are informed that communication is allowed)

At the beginning of each round, before your voting decision and your decision of how much you will extract, you will have 5 minutes to discuss the exercise with the other members of your group. During these 5 minutes, you will be able to discuss with them anything related with the exercise. Before the discussion round begins, turn your calculations sheet upside down and leave it on the table. After 5 minutes, the discussion will come to an end and you will return to your seat to decide privately what your level of extraction decision will be. 